\title{
A Maturidade da Gestão do Conhecimento Organizacional: o caso das plantas de uma organização de pesquisa
}

\author{
The Maturity of the Management of Organizational Knowledge: the case of the plants of a \\ research organizational
}

\author{
Carlos Roberto Muniz ${ }^{1}$, André Yves Cribb ${ }^{1}$
}

${ }^{1}$ Universidade Federal Rural do Rio de Janeiro, Rio de Janeiro, Brasil.

I N F O A R T I G O

Palavras-chave:

Modelo de Maturidade,

Gestão do Conhecimento,

Embrapa Agroindústria de

Alimentos.

\section{A R T I C LEINFO}

\section{Keywords:}

Maturity Model,

Knowledge Management,

Embrapa Agroindústria de

Alimentos.

\begin{abstract}
RES U M O
Muitos modelos de maturidade da Gestão do Conhecimento (GC) foram criados e até testados, porém poucos tiveram a sua aplicação publicada academicamente. Por conta dessa ausência de registros, as organizações têm dificuldades de comparar os seus resultados com outras de características semelhantes. Após o estudo bibliográfico, por aderência dos itens avaliados com os que são aplicados na Embrapa Agroindústria de Alimentos, o modelo de Oliveira e Pedron (2014) foi escolhido e aplicado nessa unidade. O instrumento, adaptado do modelo selecionado, foi respondido por 47 colaboradores, todos criam e transformam o ativo conhecimento dentro da unidade estudada. Os dados coletados foram tratados com análises estatísticas descritivas através do software Excel. A limitação deste estudo está na aplicação do modelo selecionado em uma única unidade da Empresa Brasileira de Pesquisa Agropecuária (Embrapa). Contudo, a unidade tem cinco plantas e por isso foi viável às análises quantitativas realizadas.

A B S T R A C T
Many models of maturity of knowledge management were created and even
tested, but few had their application published academically. Because of this
lack of records, organizations have difficulty comparing their results with
others with similar characteristics. After the bibliographic study, by adhesion
of the evaluated items with those that are applied in the Embrapa Agroindústria
de Alimentos, the model of Oliveira e Pedron 2014 was chosen and applied in
this unit. The instrument, adapted from the selected model, was answered by
47 collaborators. Everyone created and transformed the active knowledge
within the studied unit. The data collected were included with descriptive
statistical analyzes using Excel software. The limitation of this study lies in
the application of the selected model in a single unit of the Empresa
Brasileira de Pesquisa Agropecuária (Embrapa). However, the unit has five
plants and, therefore, was feasible for the quantitative analysis performed.
\end{abstract}

This work is licensed under a Creative Commons Attribution 4.0 International License.

Correspondência para autores:

carlos-muniz@hotmail.com (Muniz, C.R.) (ORCID: 0000-0001-8069-9226),

andre.cribb@embrapa.br (Cribb, A.Y.) (ORCID: 0000-0003-4243-394X)

DOI: doi.org/10.51359/1679-1827.2021.243003 


\section{INTRODUÇÃO}

No mundo atual, as transformações e as mudanças no dia a dia na sociedade e nas organizações acontecem com grande velocidade e o conhecimento é um recurso vital para essa transição, o avanço da era industrial para a era das redes e da informação é um exemplo (TERRA, 2000).

A GC é imperativa e decisiva nos resultados objetivados nas organizações. Por isso, ela tem em sua proposta um conjunto de práticas, ações e processos atribuídos e aplicados em indivíduos, grupos ou estruturas físicas, (ALAVI; LEIDNER,2001). Para melhorar a eficiência em organizações públicas ou privadas, a GC precisa de ações estratégicas que visem criar, armazenar, distribuir e utilizar os conhecimentos (VALENTIM, 2002)

Nesse sentido, empresas de pesquisa e desenvolvimento (P\&D), de modo geral, buscam desenvolver novos produtos ou propor soluções sistematizadas, visando a sinergia com as expectativas dos clientes (COOPER; EDGETT, 2005).

Para aumentar a efetividade dos processos de GC nas organizações foram desenvolvidos modelos de maturidade da GC, que podem mensurar a maturidade da GC no momento da avaliação, apresentar as oportunidades e pontuar a evolução dos processos da GC nas corporações (CHEN et al., 2009; LEE et al., 2005). Esses modelos podem definir os processos da GC em cinco níveis. (SERNA, 2012).

Ao longo das últimas décadas, muitos modelos de maturidade da GC foram propostos para avaliar a GC, entretanto, existem poucos relatos da aplicação dessas ferramentas (OLIVEIRA et al.,2014). A ausência de pesquisas, que aplicam os modelos de maturidade de GC, gera um hiato de dados para consulta e comparação de resultados (ARIAS, DURANGO; 2015). Esta pesquisa visa gerar dados através da aplicação de um modelo de maturidade de GC em uma organização de pesquisa e desenvolvimento

Neste estudo, com base nessas considerações, torna-se evidente o questionamento: Existe diferença de resultados ao aplicar um modelo de maturidade de GC nas plantas de uma unidade de pesquisa e desenvolvimento? Buscando responder a essa questão, este trabalho tem como objetivo comparar a maturidade da GC nas plantas de uma unidade de pesquisa e desenvolvimento. A organização onde foi aplicada o modelo de maturidade da GC é a Embrapa Agroindústria de Alimentos, maiores detalhes estão na metodologia desta pesquisa.

Este estudo está dividido em cinco seções. Após esta introdução está o referencial teórico, onde foram definidos através de fontes bibliográficas os conceitos e o modelo utilizado nesta pesquisa. Na terceira parte, está a metodologia utilizada na geração deste trabalho. $\mathrm{Na}$ quarta parte estão apresentados os resultados, as discussões e a aplicação do modelo em uma organização de pesquisa. Na quinta parte estão as considerações finais e na sexta e última seção estão as referências.

\section{REFERENCIAL TEÓRICO}

\subsection{GESTÃO DO CONHECIMENTO EM P\&D}

A GC é um processo que auxilia no desenvolvimento e aplicação do conhecimento em uma organização (SPEND, 2001). Com a GC é possível adquirir, organizar, manter, aplicar, distribuir, publicar e recriar conhecimento explícito. (ALAVI; LEIDNER, 2001). A GC consiste em um processo de criação, divulgação e uso de conhecimento visando atingir os objetivos da organização (DAVENPORT; PRUSAK, 2003).

Em uma organização de pesquisa e desenvolvimento (P\&D), o objetivo é apresentar soluções tecnológicas e/ou gerenciais para seus clientes e/ou benificiário, voltando seu foco para a sua atuação direcionada para o atendimento de demandas internas e externas (CRIBB, 2010). Para melhorar a cooperação e interação entre pesquisadores e clientes, a GC pode potencializar e desenvolver atividades e projetos (LIMA; AMARAL, 2008).

Nesse contexto, os procedimentos para a construção de estratégias de GC em organizações de P\&D 
podem ser aplicados nas seguintes etapas: 1) coordenação da equipe de trabalho; 2) análise dos ambientes (externo e interno) da organização; 3) caracterização da estratégia de gestão do negócio da organização; 4) formulação da estratégia de GC da organização; 5) Articulação e comunicação entre os stakeholders da organização (CRIBB,

2010).

As ações de GC necessitam de uma avaliação de pontos e situações que permitam apresentar os pontos fortes e fracos da organização (LIN, 2007; OLIVEIRA et al., 2011; LIN, 2011).

Com a proposta de preencher essa lacuna, surgiu o modelo de maturidade da GC, que permite uma avaliação do seu nível no momento em que é aplicada, podendo servir como ponto de partida para discussão e desenvolvimento de um plano de GC. (BATISTA,2012).

\section{ESCOLHA DO MODELO APLICADO}

O modelo de maturidade da GC, desde os primeiros, tem a função de medir o nível do gerenciamento do conhecimento e, assim, assumindo que quanto mais GC, melhor será a inovação e o resultado da corporação (PEE; KANKANHALLI, 2009). A GC pode identificar e alavancar o conhecimento coletivo em uma organização (BARÃO et al, 2017)

Esses modelos de maturidade, através de sua aplicação, podem definir que uma organização está em níveis que variam de iniciais até níveis mais avançados de GC (NGAI et al., 2013). O nível de maturidade de uma organização é definido através da avaliação dos fatores chaves que impactam na GC (OLIVEIRA et al., 2011).

No quadro 1 estão alguns modelos de maturidade da GC e autores que utilizam esses fatores para medir a maturidade da GC.

\begin{tabular}{|c|c|c|c|c|c|c|c|c|c|c|c|c|c|c|}
\hline Fatores chaves & 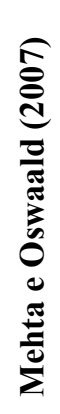 & 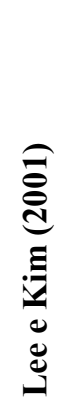 & 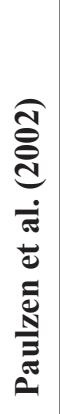 & 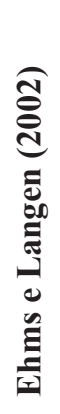 & 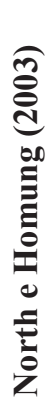 & 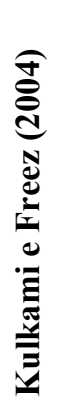 & 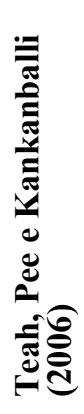 & 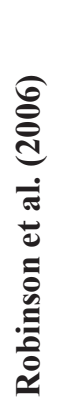 & 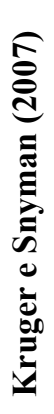 & 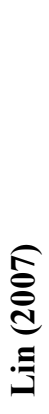 & 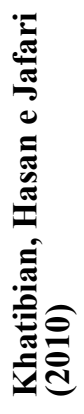 & 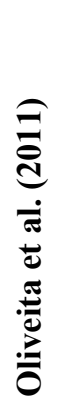 & 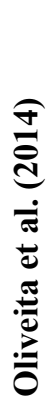 & 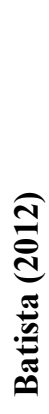 \\
\hline Clientes & & $\mathrm{X}$ & $\mathrm{X}$ & $\mathrm{X}$ & & & & & $\mathrm{X}$ & $\mathrm{X}$ & & $\mathrm{X}$ & $\mathrm{X}$ & \\
\hline Fornecedores & & $\mathrm{X}$ & & $\mathrm{X}$ & & & & & $\mathrm{X}$ & & & $\mathrm{X}$ & $\mathrm{X}$ & \\
\hline Parceiros & & $\mathrm{X}$ & & $\mathrm{X}$ & & & & & $\mathrm{X}$ & & & $\mathrm{X}$ & $\mathrm{X}$ & \\
\hline $\begin{array}{l}\text { Compartilhar o } \\
\text { Conhecimento }\end{array}$ & & $\mathrm{X}$ & & & & & & & $\mathrm{X}$ & & & $X$ & $X$ & \\
\hline Legislação & & & & & & & & & & & & $\mathrm{X}$ & & $\mathrm{X}$ \\
\hline Cultura & $\mathrm{X}$ & & & $\mathrm{X}$ & & $\mathrm{X}$ & $\mathrm{X}$ & $X$ & $\mathrm{X}$ & $\mathrm{X}$ & $\mathrm{X}$ & $\mathrm{X}$ & & $\mathrm{X}$ \\
\hline Estrutura & & $\mathrm{X}$ & & $\mathrm{X}$ & & & & & & & $\mathrm{X}$ & $\mathrm{X}$ & & $\mathrm{X}$ \\
\hline
\end{tabular}




\begin{tabular}{|c|l|l|l|l|l|l|l|l|l|l|l|l|l|l|}
\hline $\begin{array}{c}\text { Suporte da Alta } \\
\text { Administração }\end{array}$ & & & & $\mathrm{X}$ & & $\mathrm{X}$ & & & & $\mathrm{X}$ & $\mathrm{X}$ & $\mathrm{X}$ & $\mathrm{X}$ & $\mathrm{X}$ \\
\hline $\begin{array}{c}\text { Alinhamento com os } \\
\text { objetivos de negócio }\end{array}$ & $\mathrm{X}$ & & & $\mathrm{X}$ & & & $\mathrm{X}$ & $\mathrm{X}$ & $\mathrm{X}$ & & $\mathrm{X}$ & $\mathrm{X}$ & & $\mathrm{X}$ \\
\hline Objetivos da GC & $\mathrm{X}$ & $\mathrm{X}$ & & $\mathrm{X}$ & & $\mathrm{X}$ & & $\mathrm{X}$ & $\mathrm{X}$ & $\mathrm{X}$ & $\mathrm{X}$ & $\mathrm{X}$ & & $\mathrm{X}$ \\
\hline Benefícios & & & & & & & $\mathrm{X}$ & $\mathrm{X}$ & $\mathrm{X}$ & $\mathrm{X}$ & & $\mathrm{X}$ & & $\mathrm{X}$ \\
\hline Conhecimento tácito & $\mathrm{X}$ & & & & & $\mathrm{X}$ & & & $\mathrm{X}$ & & & $\mathrm{X}$ & $\mathrm{X}$ & $\mathrm{X}$ \\
\hline Conhecimento explícito & & & & & & $\mathrm{X}$ & & $\mathrm{X}$ & $\mathrm{X}$ & & & $\mathrm{X}$ & $\mathrm{X}$ & $\mathrm{X}$ \\
\hline Conhecimento crítico & & & & & & & & & $\mathrm{X}$ & & $\mathrm{X}$ & $\mathrm{X}$ & & $\mathrm{X}$ \\
\hline Tecnologia & $\mathrm{X}$ & & $\mathrm{X}$ & $\mathrm{X}$ & $\mathrm{X}$ & $\mathrm{X}$ & $\mathrm{X}$ & & $\mathrm{X}$ & $\mathrm{X}$ & $\mathrm{X}$ & $\mathrm{X}$ & & $\mathrm{X}$ \\
\hline Líder em GC & & & & & & & $\mathrm{X}$ & $\mathrm{X}$ & & $\mathrm{X}$ & & $\mathrm{X}$ & & $\mathrm{X}$ \\
\hline Sistema de recompens & $\mathrm{X}$ & & $\mathrm{X}$ & & & $\mathrm{X}$ & $\mathrm{X}$ & $\mathrm{X}$ & & $\mathrm{X}$ & $\mathrm{X}$ & $\mathrm{X}$ & & $\mathrm{X}$ \\
\hline $\begin{array}{c}\text { Criação/ } \\
\text { Armazenamento do } \\
\text { conhecimento }\end{array}$ & & & & & & & & & & & & & & \\
\hline Treinamento & $\mathrm{X}$ & & & & & $\mathrm{X}$ & $\mathrm{X}$ & & & $\mathrm{X}$ & $\mathrm{X}$ & $\mathrm{X}$ & & $\mathrm{X}$ \\
\hline Fases do processo & $\mathrm{X}$ & & $\mathrm{X}$ & $\mathrm{X}$ & $\mathrm{X}$ & $\mathrm{X}$ & $\mathrm{X}$ & $\mathrm{X}$ & $\mathrm{X}$ & $\mathrm{X}$ & $\mathrm{X}$ & $\mathrm{X}$ & & $\mathrm{X}$ \\
\hline Comunicação & & $\mathrm{X}$ & & $\mathrm{X}$ & & $\mathrm{X}$ & & $\mathrm{X}$ & & $\mathrm{X}$ & $\mathrm{X}$ & $\mathrm{X}$ & & $\mathrm{X}$ \\
\hline Orçamento & & $\mathrm{X}$ & & $\mathrm{X}$ & & & $\mathrm{X}$ & $\mathrm{X}$ & & $\mathrm{X}$ & & $\mathrm{X}$ & & $\mathrm{X}$ \\
\hline Setor público & & & & & & & & & & & & & & $\mathrm{X}$ \\
\hline
\end{tabular}

Quadro 1 - Quadro de análise comparativa entre modelos de maturidade de GC

Fonte: Oliveira et al. (2011, p.15) ampliado por Balbino, Nunes e Queiroz (2016, p.86).

O modelo de Oliveira e Pedron 2014, após uma breve análise, tem aderência nos seus itens avaliados e medidos com as atividades aplicadas e gerenciadas na organização escolhida como caso de estudo, ou seja, na Embrapa Agroindústria de Alimentos. Essa convergência, do que se aplica na organização de pesquisa e desenvolvimento estudada com dos itens medidos nesse modelo, foi determinante para escolha e aplicação do modelo na unidade de Oliveira e Pedron 2014 nesta pesquisa.

O modelo de Oliveira e Pedron (2014) foi desenvolvido a partir do modelo proposto em 2011. Ele foi criado com o objetivo de atender a uma lacuna na literatura de um modelo de maturidade e para ser aplicado em diversas áreas, organizações e países (OLIVEIRA, PEDRON;2014).

Os fatores chaves influenciam no gerenciamento dos processos de GC em uma organização (OLIVEIRA; PEDRON,2014). Pettigrew (1987) foi um dos primeiros pesquisadores a identificar e apresentar os fatores chaves contexto, conteúdo e processo. Os impactos de questões que se correlacionem com contexto, conteúdo e processo podem influenciar na gestão das corporações (PETTIGREW, 1987).

Oliveira e Pedron (2014) apresentam quatro fatores chaves no modelo de maturidade que desenvolveram, são eles: contexto interno $(\mathrm{CI})$, contexto externo $(\mathrm{CE})$, conteúdo $(\mathrm{C})$ e processo $(\mathrm{P})$ e construtos relacionados a esses fatores são definidos e apresentados por diversos autores.

Oliveira et al., (2011) apresentam e descrevem cada construto que compõe o modelo, segue: Suporte da alta administração é a relação entre a alta administração e a gestão do conhecimento; Tecnologia da Informação: Tecnologia de informação utilizada nos projetos de GC; Clientes: Participação dos clientes na gestão do conhecimento da organização; Parceiros: Participação dos parceiros na gestão do conhecimento da organização; Fornecedores: Participação dos fornecedores na gestão do conhecimento da organização; Concorrentes: 
Participação dos concorrentes na gestão do conhecimento da organização; Conhecimento Tácito: Processos e tecnologias para contemplar o conhecimento tácito da organização; Conhecimento Explícito: Processos e tecnologias para contemplar o conhecimento explícito da organização; Criação/Armazenamento: Definição das atividades organizacionais de criação e de armazenamento do conhecimento; Compartilhamento: Definição das atividades organizacionais de compartilhamento do conhecimento.

\section{Material e métodos}

A pesquisa foi desenvolvida com métodos quantitativos e com características descritivas uni e bivariada (CREWELL,2014). Através dos levantamentos de dados bibliográficos foi definido e escolhido o modelo de maturidade de GC utilizado e a organização analisada nesta pesquisa.

A Embrapa, que é organização de pesquisa e desenvolvimento, tem o conhecimento como seu principal ativo. A aplicação do modelo de maturidade da GC de Oliveira e Pedron (2014) permitiu uma avaliação dos processos de GC utilizados nas Plantas da unidade.

Ela tem, em sua composição, quarenta e duas unidades distribuídas por todas as regiões do país voltadas para pesquisa e desenvolvimento (EMPRESA BRASILEIRA DE PESQUISA AGROPECUÁRIA, 2018). Por questões de logística e acesso, a unidade escolhida foi a Embrapa Agroindústria de Alimentos (CTAA), que fica situada no bairro de Guaratiba- Rio de janeiro.

A Embrapa Agroindústria de Alimentos possui setores administrativos, setores de apoio, laboratórios e plantas. (EMPRESA BRASILEIRA DE PESQUISA AGROPECUÁRIA, 2018). Para selecionar apenas os colaboradores que manuseiam e produzem conhecimento, nesta pesquisa, o modelo de Oliveira e Pedron (2014) foi aplicado nas plantas de pesquisa e desenvolvimento da unidade. Ela possui em suas dependências cinco plantas de pesquisas e elas são nomeadas com os algarismos romanos I, II, III, IV e V. Em suas dependências, as plantas têm um total de trinta pesquisadores, dez analistas e quinze técnicos, que trabalham com pesquisa e desenvolvi- mento (EMPRESA BRASILEIRA DE PESQUISA AGROPECUÁRIA, 2018).

As opiniões dos pesquisadores, analistas e técnicos foram coletadas através do questionário adaptado do modelo de Oliveira e Pedron (2014), com o auxílio da ferramenta do Google drive ferramentas. O questionário foi enviado através de e-mail e coletado através de visitas a unidade com aqueles que manuseiam e transformam o ativo conhecimento na unidade. As questões têm como possibilidade de respostas que variam de concordo totalmente a discordo totalmente na escala Likert, que na análise dos dados variaram de 5 a 1 para obter a média de cada variável.

A população desta pesquisa consistiu no total de trinta pesquisadores, dez analistas e quinze técnicos. Um total de cinquenta e cinco pessoas, que desenvolvem conhecimento dentro da unidade. Este estudo restringiu-se a colaboradores que trabalham diretamente com pesquisa e desenvolvimento nas plantas. Já que os setores administrativos e setores de apoio têm atividades diversas, que pouco contribuiriam para as análises dos dados e da comparação dos resultados.

A amostra tem vinte e sete pesquisadores, oito analistas e doze técnicos. Foram quarenta e sete questionários analisados. A planta I teve nove respondentes, a planta II teve sete respondentes, a planta III teve doze respondentes, a planta IV teve dez respondentes e a planta V teve nove respondentes. Do total dos respondentes 72 \% apresentam no mínimo, pós-Graduação Stricto Sensu (Mestrado) Cursando ou Completo e $96 \%$ dos respondentes estão há mais de 5 anos na unidade. No próximo capitulo estão os resultados e a discussão dos dados coletados.

\section{RESULTADOS E DISCUSSÃO}

Oliveira e Pedron (2014) apresentam os quatro fatores chaves, entre eles estão o fator chave o processo - P definido por Choi e Lee em 2003 e os fatores chaves conteúdo - C e contexto interno - CI que são definidos por Oliveira e Pedron em 2011. O fator chave contexto externo - CE definido por Lin, 2007. Os construtos relacionados a esses fatores chaves são definidos e apresentados por esses autores. Cada fator chave apresenta construtos a serem avaliados e medidos na aplicação do modelo. 


\subsection{Fator chave Processo}

No quadro 2 estão as variáveis que compõem os construtos Criação/ Armazenamento e compartilhamento. Essas questões foram respondidas e tabuladas para aferição do Fator chave Processo na unidade estudada e fazem parte do modelo de Oliveira e Pedron (2014).

\begin{tabular}{|l|l|}
\hline Item & Criação/ Armazenamento (CA) \\
\hline CA_1 & Os processos ou ferramentas para criar conhecimento são definidos. \\
\hline CA_2 & Os funcionários participam frequentemente de atividades para criar conhecimento. \\
\hline CA_3 & Os funcionários frequentemente conduzem atividades de criação de conhecimento. \\
\hline CA_4 & Os processos ou ferramentas para o armazenamento do conhecimento são definidos. \\
\hline CA_5 & Os funcionários participam frequentemente de atividades para o armazenamento de conhecimento. \\
\hline CA_6 & Os funcionários frequentemente conduzem atividades para o armazenamento de conhecimento. \\
\hline & Compartilhamento (CC) \\
\hline CC_1 & $\begin{array}{l}\text { Os funcionários solicitam aos colegas que compartilhem suas habilidades quando precisam aprender } \\
\text { alguma coisa. }\end{array}$ \\
\hline CC_2 & Os funcionários gostam de ser informados sobre o que seus colegas sabem. \\
\hline CC_3 & $\begin{array}{l}\text { Os funcionários solicitam aos colegas que compartilhem suas habilidades quando precisam aprender } \\
\text { alguma coisa. }\end{array}$ \\
\hline CC_4 & Quando um funcionário é bom em alguma coisa, os colegas pedem para que os ensine a fazer. \\
\hline CC_5 & Quando os funcionários aprendem algo novo, eles compartilham o assunto com seus colegas. \\
\hline CC_6 & Os funcionários compartilham os conhecimentos que possuem com seus colegas. \\
\hline CC_7 & Os funcionários consideram importante que seus colegas saibam o que eles estão fazendo. \\
\hline CC_8 & Os funcionários compartilham com seus colegas o que eles estão fazendo. \\
\hline
\end{tabular}

Fonte: Adaptado de Oliveira Pedron (2014)

Nas tabelas 1 e 2 estão os resultados coletados na pesquisa dos construtos criação e armazenamento (CA e compartilhamento de conhecimento (CC). Neles estão a média de cada variável que compõe o modelo e a amplitude dos resultados das variáveis. Esses dados apresentam que a variável CA 4 tem maior amplitude com 1,65 e a variável CC 1 tem a menor amplitude com 0,39. Os construtos Compartilhamento do Conhecimento e Criação e Armazenamento têm em suas variáveis questões que medem o comportamento dos respondentes, esses que possuem vivências e experiências distintas, apesar de terem atividades, estruturas e perfis semelhantes. Isso pode ter influenciado nas amplitudes do resultado das plantas.

\begin{tabular}{|c|c|c|c|c|c|c|}
\hline Variável & Planta I & Planta II & Planta III & Planta VI & Planta V & Amplitude \\
\hline CA 1 & 3,56 & 3,71 & 3,17 & 3,90 & 3,22 & 0,73 \\
\hline CA 2 & 2,78 & 4,14 & 3,17 & 3,40 & 3,00 & 1,36 \\
\hline CA 3 & 2,78 & 3,57 & 3,50 & 3,60 & 3,22 & 0,82 \\
\hline CA 4 & 3,33 & 4,57 & 2,92 & 3,80 & 2,78 & 1,65 \\
\hline CA 5 & 2,89 & 3,43 & 3,00 & 3,20 & 2,33 & 1,10 \\
\hline CA 6 & 2,44 & 4,14 & 3,08 & 3,80 & 3,11 & 1,36 \\
\hline Total & $\mathbf{2 , 9 6}$ & $\mathbf{3 , 9 3}$ & $\mathbf{3 , 1 4}$ & $\mathbf{3 , 6 2}$ & $\mathbf{2 , 9 4}$ & $\mathbf{0 , 9 9}$ \\
\hline
\end{tabular}

Tabela 1: Construto Criação e armazenamento (CA)

Fonte: Elaborado pelos autores 


\begin{tabular}{|c|c|c|c|c|c|c|}
\hline Variável & Planta I & Planta II & Planta III & Planta VI & Planta V & Amplitude \\
\hline CC 1 & 4,22 & 4,00 & 3,83 & 4,00 & 4,00 & 0,39 \\
\hline CC 2 & 3,22 & 4,14 & 4,17 & 3,20 & 3,67 & 0,97 \\
\hline CC 3 & 3,89 & 3,71 & 4,00 & 4,00 & 3,89 & 0,29 \\
\hline CC 4 & 3,11 & 3,71 & 3,58 & 3,50 & 3,67 & 0,60 \\
\hline CC 5 & 2,56 & 3,71 & 3,17 & 2,90 & 2,56 & 1,15 \\
\hline CC 6 & 3,22 & 4,00 & 3,58 & 2,90 & 3,22 & 1,10 \\
\hline CC 7 & 2,89 & 4,14 & 3,67 & 4,00 & 3,22 & 1,25 \\
\hline CC 8 & 2,44 & 3,71 & 3,42 & 3,50 & 3,11 & 1,27 \\
\hline Total & $\mathbf{3 , 1 9}$ & $\mathbf{3 , 8 9}$ & $\mathbf{3 , 6 8}$ & $\mathbf{3 , 5 0}$ & $\mathbf{3 , 4 2}$ & $\mathbf{0 , 7 3}$ \\
\hline
\end{tabular}

Tabela 2: Compartilhamento de conhecimento (CC)

Fonte: Elaborado pelos autores

\subsection{Fator chave Conteúdo}

O quadro 3 são apresentadas as variáveis que compõem o fator chave Conteúdo e seus construtos. Que com a tabulação das respostas chegou-se aos valores abaixo apresentados.

\begin{tabular}{|l|l|}
\hline Item & Conhecimento Explicito \\
\hline CE_1 & $\begin{array}{l}\text { O conhecimento (como fazer, habilidades técnicas ou métodos para solucionar problemas) é bem } \\
\text { documentado. }\end{array}$ \\
\hline CE_2 & O conhecimento pode ser facilmente obtido através de documentos e manuais. \\
\hline CE_3 & Os resultados de projetos e reuniões são documentados. \\
\hline CE_4 & O conhecimento é compartilhado através de documentos como, por exemplo, manuais. \\
\hline & Conhecimento Tácito \\
\hline CT_1 & O meu conhecimento pode facilmente ser obtido com especialistas ou colegas. \\
\hline CT_2 & É fácil obter face-a-face conselhos de especialistas. \\
\hline CT_3 & Conversas informais e reuniões são utilizadas para compartilhar conhecimento. \\
\hline CT_4 & O conhecimento é obtido através do relacionamento entre colegas. \\
\multicolumn{2}{|c}{ Quadro 3: Variáveis do fator-chave Conteúdo } \\
\end{tabular}

Nas tabelas 3 e 4 estão os resultados coletados na pesquisa dos construtos conhecimento explícito (CE) e conhecimento tácito (CT). Neles estão a média de cada variável que compõe o modelo e a amplitude dos resultados das variáveis. A variável CT1 tem a maior amplitude com 2,13 e CT3 tem a menor amplitude de 0,36. Ambos os construtos, Conhecimento Explícito (CE) e Conhecimento (CT), tiveram a amplitude de 1,11. No Conhecimento Tácito (CT), que teve como destaque a variável CT $1 \mathrm{com}$ 2,13 de amplitude nos resultados das plantas. Esse resultado pode demostrar que em algumas plantas o conhecimento pode ser acessado por outros colegas ou especialistas e em outras plantas o acesso pode ter alguma dificuldade de acesso ao conhecimento produzido. No construto Conhecimento Explícito (CE) todas as variáveis tiveram a amplitude acima de 1,00, pode-se interpretar, em algumas plantas, que existe uma dificuldade de acesso à repositórios de conhecimento. A seguir estão os dados dos construtos do fator chave conteúdo. 


\begin{tabular}{|c|c|c|c|c|c|c|}
\hline Variável & Planta I & Planta II & Planta III & Planta VI & Planta V & Amplitude \\
\hline CE 1 & 3,67 & 4,29 & 3,58 & 3,50 & 3,22 & 1,07 \\
\hline CE 2 & 3,89 & 4,14 & 3,58 & 3,50 & 3,11 & 1,03 \\
\hline CE 3 & 3,56 & 4,57 & 4,08 & 4,30 & 3,44 & 1,13 \\
\hline CE 4 & 3,67 & 4,43 & 3,50 & 3,70 & 3,22 & 1,21 \\
\hline Total & $\mathbf{3 , 6 9}$ & $\mathbf{4 , 3 6}$ & $\mathbf{3 , 6 9}$ & $\mathbf{3 , 7 5}$ & $\mathbf{3 , 2 5}$ & $\mathbf{1 , 1 1}$ \\
\hline
\end{tabular}

Tabela 3: Conhecimento Explícito (CE)

Fonte: Elaborado pelo autor

\begin{tabular}{|c|c|c|c|c|c|c|}
\hline Variável & Planta I & Planta II & Planta III & Planta VI & Planta V & Amplitude \\
\hline CT 1 & 3,56 & 4,57 & 3,52 & 2,90 & 2,44 & 2,13 \\
\hline CT 2 & 3,78 & 4,43 & 3,17 & 3,60 & 2,78 & 1,65 \\
\hline CT 3 & 3,78 & 4,14 & 4,08 & 4,00 & 3,78 & 0,36 \\
\hline CT 4 & 3,78 & 3,89 & 3,67 & 3,40 & 3,56 & 0,49 \\
\hline Total & $\mathbf{3 , 7 2}$ & $\mathbf{4 , 2 5}$ & $\mathbf{3 , 1 7}$ & $\mathbf{3 , 4 8}$ & $\mathbf{3 , 1 4}$ & $\mathbf{1 , 1 1}$ \\
\hline
\end{tabular}

Tabela 4: Conhecimento Tácito (CT)

Fonte: Elaborado pelos autores

5.3. Fator chave Contexto Interno (CI).

As variáveis do Fator chave Contexto interno e seus respectivos construtos estão no quadro 4. Essas questões foram utilizadas para a coleta de dados e tabulação dos resultados apresentados.

\begin{tabular}{|l|l|}
\hline Item & Suporte da Alta Direção (SA) \\
\hline SA_1 & $\begin{array}{l}\text { A alta administração acredita que estimular o compartilhamento de conhecimento entre os } \\
\text { funcionários é benéfico. }\end{array}$ \\
\hline SA_2 & $\begin{array}{l}\text { A alta administração sempre estimula os funcionários a compartilharem seus conhecimentos com } \\
\text { os colegas. }\end{array}$ \\
\hline SA_3 & $\begin{array}{l}\text { A alta administração fornece a maioria dos recursos necessários para que os funcionários } \\
\text { compartilhem conhecimento. }\end{array}$ \\
\hline SA_4 & $\begin{array}{l}\text { A alta administração se empenha para ver os funcionários satisfeitos em compartilharem seus } \\
\text { conhecimentos com os colegas. }\end{array}$ \\
\hline & Tecnologia da Informação (TI) \\
\hline TI_1 & Os funcionários usam armazenamento eletrônico para acessar conhecimento. \\
\hline
\end{tabular}




\begin{tabular}{|l|l|}
\hline TI_2 & $\begin{array}{l}\text { Os funcionários usam redes de conhecimento (por exemplo, comunidades virtuais, groupware) para } \\
\text { se comunicar. }\end{array}$ \\
\hline TI_3 & $\begin{array}{l}\text { A tecnologia utilizada permite que os funcionários compartilhem conhecimento com pessoas de } \\
\text { outras empresas. }\end{array}$ \\
\hline TI_4 & $\begin{array}{l}\text { A tecnologia utilizada permite que os funcionários compartilhem conhecimento com pessoas da } \\
\text { própria empresa }\end{array}$ \\
\hline
\end{tabular}

Quadro 4: Variáveis do fator-chave Contexto interno

Fonte: Adaptado de Oliveira Pedron (2014)

Nas tabelas 5 e 6 estão os resultados coletados na pesquisa dos construtos suporte da alta administração (SA) e tecnologia da informação (TI). Neles estão a média e a amplitude de cada variável que compõe o modelo. As variáveis T2 com 1,99 e SA 3 com 1,30 apresentam os maiores valores de amplitude dos seus respectivos construtos e o TI 3 com o valor de 0,53 foi o resultado com menor variação dentre eles. Em análise dos resultados do construto $\mathrm{SA}$, ele teve resultados distintos entre as plantas, ainda que tenham os mesmos gestores. No construto TI, as plantas apresentam condições semelhantes em suas estruturas e ainda sim tiveram resultados distintos.

O fator chave CI demostra que mesmo com hierarquia iguais e com estruturas semelhantes, nesse fator chave, os respondentes têm percepções diferentes.

\begin{tabular}{|c|c|c|c|c|c|c|}
\hline Variável & Planta I & Planta II & Planta III & Planta VI & Planta V & Amplitude \\
\hline SA 1 & 3,56 & 4,00 & 2,92 & 3,80 & 3,89 & 1,08 \\
\hline SA 2 & 3,67 & 4,29 & 3,58 & 3,30 & 3,22 & 1,07 \\
\hline SA 3 & 2,56 & 3,86 & 3,33 & 3,00 & 3,00 & 1,30 \\
\hline SA 4 & 3,00 & 4,14 & 3,17 & 3,00 & 2,89 & 1,25 \\
\hline Total & $\mathbf{3 , 1 9}$ & $\mathbf{4 , 0 7}$ & $\mathbf{3 , 2 5}$ & $\mathbf{3 , 2 8}$ & $\mathbf{3 , 2 5}$ & $\mathbf{0 , 8 8}$ \\
\hline
\end{tabular}

Tabela 5: Suporte da alta Administração (SA)

Fonte: Elaborado pelos autores

\begin{tabular}{|c|c|c|c|c|c|c|}
\hline Variável & Planta I & Planta II & Planta III & Planta VI & Planta V & Amplitude \\
\hline TI 1 & 4,56 & 4,57 & 4,08 & 4,20 & 3,56 & 1,01 \\
\hline TI 2 & 3,11 & 4,43 & 3,17 & 4,10 & 2,44 & 1,99 \\
\hline TI 3 & 3,33 & 3,86 & 3,17 & 3,70 & 2,78 & 0,53 \\
\hline TI 4 & 4,22 & 4,14 & 3,75 & 4,30 & 3,33 & 0,97 \\
\hline Total & $\mathbf{3 , 8 1}$ & $\mathbf{4 , 2 5}$ & $\mathbf{3 , 5 4}$ & $\mathbf{4 , 0 8}$ & $\mathbf{3 , 0 3}$ & $\mathbf{1 , 2 2}$ \\
\hline
\end{tabular}

Tabela 6: Tecnologia da Informação (TI)

Fonte: Elaborado pelos autores

\subsection{Fator chave Contexto Externo (CE)}

No quadro 5 estão as variáveis adaptadas do modelo de Oliveira e Pedron (2014), que compõem o fator chave Contexto externo e seus respectivos construtos. 


\begin{tabular}{|l|l|}
\hline Item & Variável \\
\hline CL_1 & O conhecimento é compartilhado com os nossos clientes. \\
\hline CL_2 & Os clientes são solicitados a compartilharem seus conhecimentos com a empresa. \\
\hline CL_3 & O conhecimento dos clientes é incorporado nas ações da empresa. \\
\hline & Cliente \\
\hline FO_1 & O conhecimento é compartilhado com os nossos fornecedores. \\
\hline FO_2 & Os fornecedores são solicitados a compartilharem seus conhecimentos com a empresa. \\
\hline FO_3 & O conhecimento dos fornecedores é incorporado nas ações da empresa. \\
\hline & Fornecedor \\
\hline PA_1 & O conhecimento é compartilhado com os nossos parceiros. \\
\hline PA_2 & Os nossos parceiros são solicitados a compartilharem seus conhecimentos com a empresa. \\
\hline PA_3 & O conhecimento dos parceiros é incorporado nas ações da empresa. \\
\hline & Parceiro \\
\hline
\end{tabular}

Quadro 5: Variáveis do fator-chave Contexto externo

Fonte: Adaptado de Oliveira Pedron (2014)

Nas tabelas 7, 8 e 9 estão os resultados coletados na pesquisa dos construtos Parceiro (PA), Cliente (CL) e Fornecedor (FO), respectivamente. Eles apresentam a média de cada variável que compõe o modelo e a amplitude dos resultados de cada planta.

O construtor Parceiro (PA) teve 1,20 de amplitude e a sua variável com maior amplitude com 1,37 de amplitude foi a variável PA 3, que questiona o colaborador sobre o compartilhamento do conhecimento dos parceiros com a unidade. $\mathrm{O}$ construto $\mathrm{CL}$ teve 1,01 de amplitude e a sua variável com maior amplitude foi a CL 2 com 1,39, que questiona se o cliente compartilha o seu conhecimento com a unidade. O construto FO com 0,93 de amplitude teve a variável FO 2 com a maior amplitude com 1,51, que questiona o colaborador sobre compartilhamento do conhecimento com os fornecedores com a unidade. Essas variáveis, que questionam os respondentes sobre compartilhamento de conhecimento com o ambiente externo, demonstram que o Fator chave Contexto Externo tem percepções dos respondentes distintas do relacionamento com parceiros, Fornecedores e Clientes Externos.

Como as plantas e seus colaboradores, de modo geral, relacionam-se individualmente e de forma autônoma, pode-se inferir que elas têm percepções diferentes do compartilhamento do conhecimento com os Parceiros, Fornecedores e Clientes.

\begin{tabular}{|c|c|c|c|c|c|c|}
\hline Variável & Planta I & Planta II & Planta III & Planta VI & Planta V & Amplitude \\
\hline PA 1 & 3,78 & 4,43 & 3,67 & 3,20 & 4,00 & 1,23 \\
\hline PA 2 & 3,22 & 4,29 & 3,83 & 3,30 & 3,44 & 0,99 \\
\hline PA 3 & 3,33 & 4,57 & 3,83 & 3,20 & 3,67 & 1,37 \\
\hline Total & $\mathbf{3 , 4 4}$ & $\mathbf{4 , 4 3}$ & $\mathbf{3 , 7 8}$ & $\mathbf{3 , 2 3}$ & $\mathbf{3 , 7 0}$ & $\mathbf{1 , 2 0}$ \\
\hline
\end{tabular}

Tabela 7: Parceiro (PA)

Fonte: Elaborado pelos autores

\begin{tabular}{|c|c|c|c|c|c|c|}
\hline Variável & Planta I & Planta II & Planta III & Planta VI & Planta V & Amplitude \\
\hline CL 1 & 3,78 & 4,43 & 3,67 & 3,80 & 3,22 & 1,21 \\
\hline CL 2 & 3,22 & 4,29 & 3,33 & 2,90 & 3,44 & 1,39 \\
\hline CL 3 & 3,33 & 4,00 & 3,17 & 3,00 & 3,22 & 1,00 \\
\hline Total & $\mathbf{3 , 4 1}$ & $\mathbf{4 , 2 4}$ & $\mathbf{3 , 3 9}$ & $\mathbf{3 , 2 3}$ & $\mathbf{3 , 3 0}$ & $\mathbf{1 , 0 1}$ \\
\hline
\end{tabular}

Tabela 8: Cliente (CL)

Fonte: Elaborado pelos autores 


\begin{tabular}{|c|c|c|c|c|c|c|}
\hline Variável & Planta I & Planta II & Planta III & Planta VI & Planta V & Amplitude \\
\hline FO 1 & 3,00 & 3,43 & 3,08 & 2,80 & 3,00 & 0,63 \\
\hline FO 2 & 2,89 & 4,29 & 3,00 & 3,20 & 2,78 & 1,51 \\
\hline FO 3 & 3,00 & 3,86 & 3,50 & 3,30 & 3,00 & 0,86 \\
\hline Total & $\mathbf{2 , 9 6}$ & $\mathbf{3 , 8 6}$ & $\mathbf{3 , 1 9}$ & $\mathbf{3 , 1 0}$ & $\mathbf{2 , 9 3}$ & $\mathbf{0 , 9 3}$ \\
\hline
\end{tabular}

Tabela 9: Fornecedor (FO)

Fonte: Elaborado pelos autores

Na figura 1, o gráfico radar apresenta todos os resultados dos construtos coletados nesta pesquisa. Nele podemos notar que os construtos Clientes e Suporte da Alta Administração têm os resultados das plantas I, III, IV e V quase que sobrepostos, porém a planta II apresenta uma distância dos demais resultados dos construtos. Os construtos Tecnologia da informação e Compartilhamento têm nas cinco plantas resultados que não convergem no gráfico, todos os pontos estão em posições diferentes.

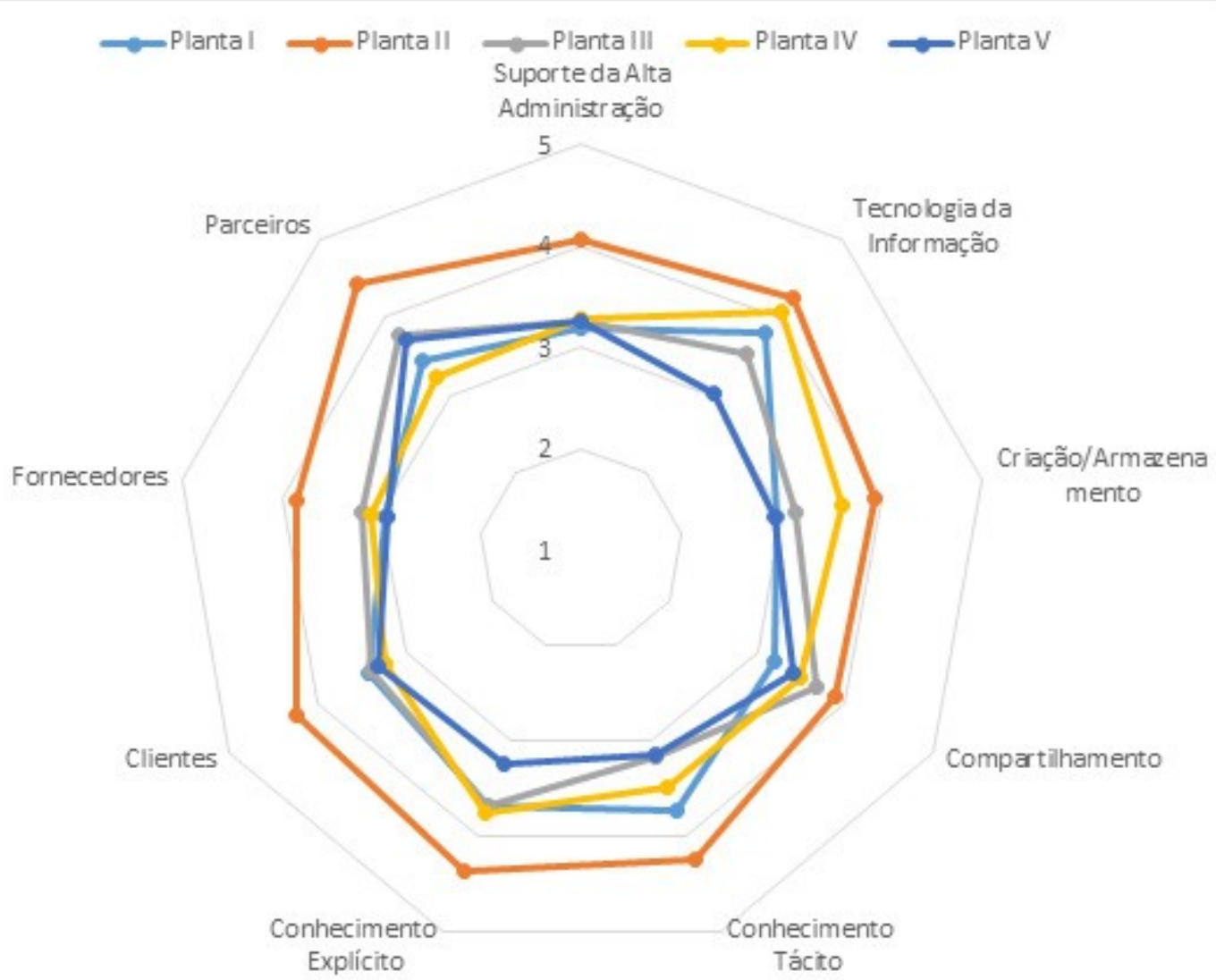

Figura 1: Gráfico radar dos resultados dos construtos nas Plantas

Fonte: Adaptado de Oliveira e Pedron 2011

Com os resultados apresentados da tabela 10 e na figura 1 pode-se afirmar, conforme o Oliveira e Pedron (2011) que o nível de maturidade da planta I da Embrapa Agroindústria de Alimentos atingiu o nível 4 e as demais plantas está no nível 3. Segundo Achive, 2018, pode existir uma lacuna de conhecimento entre o que se acredita ser o problema, com o que realmente acontece, isso pode dificultar as ações e comunicações. 


\begin{tabular}{|c|c|c|c|}
\hline Fator Chave & Variável & Média & \\
\hline \multirow{2}{*}{$\begin{array}{c}\text { Contexto } \\
\text { Interno }\end{array}$} & SA - Suporte da Alta Administração & 3,44 & \multirow[t]{2}{*}{3,60} \\
\hline & TI - Tecnologia da Informação & 3,75 & \\
\hline \multirow{3}{*}{$\begin{array}{l}\text { Contexto } \\
\text { Externo }\end{array}$} & $\mathrm{CL}-$ Clientes & 3,51 & \multirow[b]{3}{*}{3,47} \\
\hline & $\mathrm{PA}$ - Parceiros & 3,73 & \\
\hline & FO - Fornecedores & 3,19 & \\
\hline \multirow{2}{*}{ Conteúdo } & CT - Conhecimento Tácito & 3,62 & \multirow[t]{2}{*}{3,64} \\
\hline & CE - Conhecimento Explícito & 3,67 & \\
\hline \multirow{2}{*}{ Processos } & CA - Criação/Armazenamento & 3,28 & \multirow[t]{2}{*}{$3, \mathbf{4 3}$} \\
\hline & CC - Compartilhamento & 3,54 & \\
\hline
\end{tabular}

Tabela 10: Resultado médio dos construtos e fatores chaves

Fonte: Elaborado pelo autor

Após a apresentação dos resultados e análises dos Fatores chaves, construtos e variáveis, a seguir estão as configurações finais dos resultados coletados.

\section{Considerações finais}

Os dados apresentados na última seção indicam que existe diferença nos resultados das plantas da Embrapa Agroindústria de Alimentos na aplicação do modelo de maturidade da GC de Oliveira e Pedron (2014). Esses resultados convergem com a pesquisa de Pee e Kankanhalli (2009), onde concluem que departamentos diferentes de uma mesma universidade podem ter resultados irregulares em áreas distintas dessa mesma organização na aferição dos processos da GC.

A aplicação do modelo de maturidade da GC de Oliveira e Pedron (2014) na Embrapa Agroindústria de Alimentos indicou que os colaboradores das plantas, ainda que as condições sejam semelhantes na estrutura das plantas e nas características dos colaboradores, tiveram percepções diferentes dos fatores chaves e construtos avaliados nesta pesquisa. Isso pode levar a uma avaliação e consequente plano de ação distorcidos da realidade e assim, direcionar a decisões equivocadas no campo gerencial. No campo acadêmico esses resultados podem levar, caso não se individualize os resultados, a conclusões distorcidas. Segundo Achive, 2018, pode existir uma lacuna de conhecimento entre o que se acredita ser o problema, com o que realmente acontece. Isso pode dificultar as ações e comunicações dentro das organizações.

Este estudo limitou-se à aplicação do modelo em uma única unidade da Empresa Brasileira de Pesquisa Agropecuária (Embrapa). Apesar disso, por conta da quantidade de plantas de pesquisa existentes nesta unidade, foi possível a aplicação do modelo proposto no estudo, não havendo prejuízos às análises quantitativas nele realizadas.

Em pesquisas futuras, a aplicação do modelo de Oliveira e Pedron (2014) em uma quantidade maior de unidades e organizações permitirá uma avaliação mais ampla das diferenças de percepções de departamentos com as mesmas condições. 


\section{REFERÊNCIAS}

ALAVI, M. and LEIDNER, D.E, “'Review: knowledge management and knowledge management systems: conceptual foundations and research issues”, MIS Quarterly, Vol. 25 No. 1, pp. 107-36, 2001.

ARIAS-PÉREZ, J. E.; DURANGO-YEPES, C. M. Exploring knowledge management maturity from funcionalist and interpretivist perspectives. Entramado, v. 11, p. 94-104, 2015.

ACHIVE, T. (2018). Coexistence of the natural and the artificial in the mind: Engineering the non-possible. Actas de Ingeniería, 4(1), 35-42.

BATISTA, F.F. Modelo de gestão do conhecimento para a administração pública brasileira: como implementar a gestão do conhecimento para produzir resultados em benefício do cidadão. Brasília: Ipea, 2012. 132p.

BARÃO, A., BRAGA DE VASCONCELOS, J., ROCHA, A., \& PEREIRA, R. (2017). A knowledge management approach to capture organizational learning networks. International Journal of Information Management, 37, 735740.

CHEN, M. Y., HUANG, M. J., \& CHENG, Y. C. (2009). Measuring knowledge management performance using a competitive perspective: An empirical study. Expert Systems with Applications, 36(4), 8449-8459.

CHOI, B., \& Lee, H. (2003). An empirical investigation of KM styles and their effect on corporate performance. Information \& Management, 40(5), 403-417.

COOPER, R. G., \& EDGETT, S. J. (2005). Lean, rapid, and profitable new product development. Canada: Product development institute.

CRESWELL, J.W. Projeto de Pesquisa: métodos qualitativo, quantitativo e misto. Porto Alegre: Artmed, 2010.

CRIBB, A. Y. Uma abordagem pragmática de construção de estratégias de gestão do conhecimento em organizações. Revista Iberoamericana de Sistemas, Cibernética e Informática, Vol. 7, No. 1, pp. 75-80, 2010.

DAVENPORT, T.; PRUSAK, L. Conhecimento empresarial. Rio de Janeiro: Campus, 2003.

EHMS, K. e LANGEN, M. (2002), «Holistic development of knowledge management with KMMM». Siemens AG, Alemanha.

EMPRESA BRASILEIRA DE PESQUISA AGROPECUÁRIA - EMBRAPA.2018. Organograma Disponível em: $<$ https://www.embrapa.br $>$. Acesso em: Novembro 2018.

KHATIBIAN, N.; HASAN, T.; JAFARI, H.A. "Measurement of knowledge management maturity level within organizations”. Business Strategy Series, v.11, n.1, p.54-70, 2010.

KRUGER, C. J.; SNYMAN, M. M. M. Formulation of a strategic knowledge management maturity model. South African Journal of Information Management, v.7, n.2, p.1-11, 2007.

KULKARNI, U.; ST LOUIS, R. Organizational self-assessment of knowledge management maturity. In: AMERICAS CONFERENCE ON INFORMATION SYSTEMS, 2004, Tampa, Florida, 2542-2551.

LEE, J.H., KIM Y.G. A stage model of organizational knowledge management: a latent content analysis. Expert Systems with Applications, v.20, n.4, p.299-311, 2001.

LIMA, K. K. de; AMARAL, D. C. Práticas de gestão do conhecimento em grupos de pesquisa da rede Instituto Fábrica do Milênio. Gestão da Produção [on-line], v. 15, n. 2, p. 291-305, 2008.

LIN, H.F. A stage model of knowledge management: an empirical investigation of process and effectiveness. Journal of Information Science, v.33, n.6, p.643-659, 2007.

LIN, H. Antecedents of the stage-based knowledge management evolution. Journal of Knowledge Management, v.15, n.1, p.136-155, 2011. 
LEE, K.C.; LEE, S.; KANG, I.W. KMPI: measuring KM performance, Information and Management, v.42, n.1, p.469-82, 2005.

LIMA, K.K.; AMARAL, D.C. Práticas de gestão do conhecimento em grupos de pesquisa da rede Instituto Fábrica do Milênio. Gest. Prod., São Carlos, v. 15, n. 2, p. 291-305, maio-ago. 2008.

NGAI, E.W.T., Chau, D.C.K., Poon, J.K.L. and To, C.K.M. (2013) 'Energy and utility management maturity model for sustainable manufacturing process', Int. J. Production Economics, Vol. 146, No. 2, pp.453-464.

NORTH, K. e HORNUNG, T. (2003), «Benefits of knowledge management: results of the German Award 'Knowledge Management 2002'». Journal of Universal Computer Science, vol. 9(6), pp. 463-471.

OLIVEIRA, M., Pedron, C. D., Nodari, F., \& Ribeiro, R. (2014, September). Knowledge management in small and micro enterprises: applying a maturity model. In European Conference on Knowledge Management (Vol. 2, p. 757). Academic Conferences International Limited.

OLIVEIRA, M., \& Pedron, C. D., Maturity Model for Knowledge Management and Strategic Benefits. European Conference on Knowledge Management, 1, 1-10, 2014.

OLIVEIRA, M., Pedron, C., Romão, M., \& Becker, G. . Proposta de um modelo de maturidade para Gestão do Conhecimento: KM3. Revista Portuguesa e Brasileira de Gestão, 10(4), 14-25, 2011.

PAULZEN, O.; DOUMI, M.; PERC, P. e CEREIJO-ROIBAS, A. (2002), «A maturity model for quality improvement in knowledge management». ACIS Proceedings.

PEE, L.G.; KANKANHALLI, A. A model of knowledge organizational management maturity: based on people, process and technology. Journal of Information and Knowledge Management, v.8, n.2, p.79-99, 2009.

PETTIGREW, Andrew M. Context and action in the transformation of the firm. Journal of Management Studies, v. 24, n. 6, p. 649-670, 1987.

ROBINSON, H. S.; ANUMBA, C. J.; CARRILlO, P. M. e AL-GHASSANI, A. M. (2006), «STEPS: a knowledge management maturity roadmap for corporate sustainability». Business Process Management Journal, vol. 12(6), pp. 793-808.

SERNA, E. Maturity model of knowledge management in the interpretivist perspective, International Journal of Information Management, v.32, n.4, p.365-371, 2012.

SPEND,J. C., Gerenciando sistemas de conhecimento. In: M. T. L. Fleury, M. M. Oliveira Jr. (orgs.), Gestão estratégica do conhecimento, Cap. 1, pp. 27- 49. São Paulo: Atlas, 2001.

TERRA, J.C.C. Gestão do Conhecimento: o grande desafio empresarial, uma abordagem baseada no aprendizado e na criatividade. São Paulo: Negócio Editora, 2000. 283p.

VALENTIM, M.L.P. Inteligência Competitiva em Organizações: dado, informação e conhecimento. DataGramaZero. Ciência da Informação, v. 3, n. 4, 2002. 